\title{
Malaria investigation and treatment of children admitted to county hospitals in western Kenya
}

Beatrice I. Amboko ${ }^{*}$, Philip Ayieko ${ }^{1}$, Morris Ogero ${ }^{1}$, Thomas Julius ${ }^{1}$, Grace Irimu ${ }^{1,2}$, Mike English ${ }^{1,3}$ and on behalf of Clinical Information Network authors

\begin{abstract}
Background: Up to $90 \%$ of the global burden of malaria morbidity and mortality occurs in sub-Saharan Africa and children under-five bear a disproportionately high malaria burden. Effective inpatient case management can reduce severe malaria mortality and morbidity, but there are few reports of how successfully international and national recommendations are adopted in management of inpatient childhood malaria.
\end{abstract}

Methods: A descriptive cross-sectional study of inpatient malaria case management practices was conducted using data collected over 24 months in five hospitals from high malaria risk areas participating in the Clinical Information Network (CIN) in Kenya. This study describes documented clinical features, laboratory investigations and treatment of malaria in children (2-59 months) and adherence to national guidelines.

Results: A total of 13,014 children had a malaria diagnosis on admission to the five hospitals between March, 2014 and February, 2016. Their median age was 24 months (IQR 12-36 months). The proportion with a diagnostic test for malaria requested was 11,981 (92.1\%). Of 10,388 patients with malaria test results documented, 8050 (77.5 \%) were positive and anti-malarials were prescribed in 6745 (83.8 \%). Malaria treatment was prescribed in 1613/2338 (69.0\%) children with a negative malaria result out of which only 52 (3.2\%) had a repeat malaria test done as recommended in national guidelines. Documentation of clinical features was good across all hospitals, but quinine remained the most prescribed malaria drug (47.2 \% of positive cases) although a transition to artesunate (46.1 \%) was observed. Although documented clinical features suggested approximately half of positive malaria patients were not severe cases artemether-lumefantrine was prescribed on admission in only $3.7 \%$ cases.

Conclusions: Despite improvements in inpatient malaria care, high rates of presumptive treatment for test negative children and likely over-use of injectable anti-malarial drugs were observed. Three years after national policy change, there is a gradual transition to artesunate. Continued efforts to support improved routine inpatient malaria care through dissemination and implementation of guidelines, and access to recommended drugs are needed together with improved capacity of hospitals to investigate other causes of severe illness in children. Efforts to improve clinical information could help track progress.

Keywords: Malaria, Under-fives, Children, Anti-malarials, Malaria case management, Inpatient

\section{Background}

Worldwide estimates indicate that three-quarters of malaria infections involving children under- 5 years occur

\footnotetext{
*Correspondence: BAmboko@kemri-wellcome.org

${ }^{1}$ Kenya Medical Research Institute/Wellcome Trust Research Programme, P.O. Box 43640, Nairobi 00100, Kenya

Full list of author information is available at the end of the article
}

in sub-Saharan Africa [1]. Malaria case management backed up with rapid diagnostics or laboratory investigations is the main strategy for treating malaria in lowincome countries [2]. The case management strategy promoted by the World Health Organization (WHO) is based on well-described clinical features of severe malaria [3-6] and effectiveness trials of recommended

\section{Biomed Central}

(C) 2016 The Author(s). This article is distributed under the terms of the Creative Commons Attribution 4.0 International License (http://creativecommons.org/licenses/by/4.0/), which permits unrestricted use, distribution, and reproduction in any medium, provided you give appropriate credit to the original author(s) and the source, provide a link to the Creative Commons license, and indicate if changes were made. The Creative Commons Public Domain Dedication waiver (http://creativecommons.org/ publicdomain/zero/1.0/) applies to the data made available in this article, unless otherwise stated. 
treatments [7]. The WHO in 2010, revised its treatment policy to recommend universal testing for all suspected malaria cases prior to treatment, and replacement of the then existing first-line anti-malarial drugs with new therapies in both outpatient and inpatient malaria treatment $[8,9]$. These recommendations were adopted in Kenya in 2010 [10] and in 2012 [11], but although reports of outpatient malaria treatment change from sulfadoxinepyrimethamine (SP) to artemisinin-based combination therapy (ACT) show major improvements in quality of malaria case management, significant challenges including drug stock-outs still persist $[12,13]$.

In the inpatient setting such assessments are rare. Existing primary studies on inpatient malaria treatment suggest a need to consider the wider clinical decisionmaking context in addition to simple dissemination of clinical guidelines [14, 15]. Similarly, systematic reviews indicate that clinical guidelines have a variable effect on uptake of new drugs and diagnostics in secondary care (equivalent to County Hospitals in Kenya). Among the factors that have been postulated to have an effect on guideline implementation especially with regard to uptake of new drugs and diagnostics is strong professional networks [16]. Descriptive analysis of inpatient childhood malaria treatment practices in Kenyan hospitals in the period following treatment guidelines amendments in Kenya was done. Data obtained from the recently established paediatric Clinical Information Network in Kenya were used to examine adoption of malaria guidelines which recommend parasitological diagnosis of malaria, adherence to test results, and use of injectable artesunate and not quinine in treatment $[9,17,18]$.

\section{Methods}

\section{Study design and setting}

This was a descriptive cross sectional study. A subset of the data from five of the 14 hospitals participating in the CIN collected during a 24-month period (March, 2014 to February, 2016) was used. All the five hospitals are located in the western Kenya, a high malaria prevalence region and serve predominantly poor populations.

\section{Participants}

All children aged between 2 and 59 months admitted to the paediatric wards in the participating hospitals with a diagnosis of malaria were eligible for inclusion in the study.

\section{Data collection and management}

Since its establishment in October 2013 CIN has supported data capture from paediatric medical records that include a standardised admission record form using the non-proprietary Research Electronic Data Capture
(REDCap) tool in all participating hospitals [17]. Support provided to hospitals participating in the network includes an additional post for a clerical assistant in each hospital and provision of one basic computer. Data collection is done on a daily basis by the clerk from medical records of all admitted patients in paediatric wards using detailed standard operating procedures under the joint supervision of the hospital medical records department and the CIN team. Data quality is promoted through the use of range and quality checks in the electronic data tool and error checking procedures. The details of data management have been previously published [17]. In brief, a process for validating the data collected at each site is conducted bimonthly through conducting double entry for selected records. During the validation at least 140 records previously entered by CIN data clerks in the 2-month period prior to the validation are re-entered by an independent trained data collection team. Ten records are selected at random for re-entry at each hospital (giving a total of 140 records across the network). Analysis is then done to validate data using $\mathrm{R}$ scripts that conduct field by field comparison for all fields in the database. A total concordance score is then calculated for each record and feedback is immediately given to data clerks in areas where there is discordance between the two sets of entries. During the most recent round of data validation the overall concordance was $85 \%$.

\section{Statistical analysis}

No formal sample size calculations were undertaken, but data on over 13,000 children aged between 2 and 59 months with an admission diagnosis of malaria were considered adequate to provide reasonably precise estimates around malaria case management practices. Descriptive analysis of the malaria admissions pooled across all hospitals and for each hospital included calculating frequencies, relative frequencies and medians (IQR) for categorical and continuous variables, respectively. Indicators of good practice for five key areas of inpatient malaria case management spanning clinical assessment, laboratory investigation and treatment are defined in Table 1 and proportions of patients managed according to the current malaria guidelines are reported. The proportion of children for whom six specific features of malaria were assessed and documented in medical records was calculated. The level of adherence to parasitological testing recommendations was determined by calculating the percentage of malaria admissions with parasitological confirmation at the time of initiating anti-malarial drugs. Finally, for parenteral anti-malarials the proportion with the correct dosages based on body weight (drug dose per $\mathrm{kg}$ body weight), allowing for the dosage to be within $20 \%$ of recommended dosage 
Table 1 Malaria policy recommendations and the indicators used to assess implementation and adherence

\begin{tabular}{|c|c|}
\hline Policy recommendation & Indicator \\
\hline $\begin{array}{l}\text { All key clinical features of severe malaria should be assessed and docu- } \\
\text { mented }\end{array}$ & $\begin{array}{l}\text { Proportion among the children with a diagnosis of malaria with } \\
\text { documented assessment of the following clinical features: level of con- } \\
\text { sciousness (AVPU scale), deep acidotic breathing or chest in-drawing } \\
\text { (respiratory distress), fever, ability to drink, convulsions and pallor }\end{array}$ \\
\hline $\begin{array}{l}\text { All suspected malaria cases without a sign of severe disease should have } \\
\text { parasitological confirmation by diagnostic testing before initiating treat- } \\
\text { ment }\end{array}$ & $\begin{array}{l}\text { Proportion of patients with a diagnosis of malaria with a malaria test } \\
\text { ordered on the admission date and with the results recorded in the } \\
\text { medical notes or laboratory register }\end{array}$ \\
\hline Malaria positive cases should be prescribed anti-malarial drug & $\begin{array}{l}\text { Proportion with malaria positive test prescribed anti-malarials } \\
\text { Proportion with malaria negative test prescribed anti-malarials } \\
\text { Type of anti-malarial drugs prescribed }\end{array}$ \\
\hline $\begin{array}{l}\text { Anti-malarial drug dosages; Quinine loading dose of } 20 \text { mg, maintenance } \\
\text { dose of } 10 \mathrm{mg} \text { and artesunate at } 2.4 \mathrm{mg} \text { per } \mathrm{kg} \text { body weight with a } 20 \% \\
\text { margin of error }\end{array}$ & $\begin{array}{l}\text { Proportion with malaria positive prescribed quinine or artesunate and } \\
\text { proportions with correct dosing }\end{array}$ \\
\hline $\begin{array}{l}\text { Management of malaria negative; anti-malarial drugs should be withheld in } \\
\text { non-severe malaria cases with a negative test; those with signs of severe } \\
\text { malaria be started on presumptive treatment but testing should be } \\
\text { repeated }\end{array}$ & $\begin{array}{l}\text { Proportion of patients with admission diagnosis of severe malaria but a } \\
\text { negative admission test and given anti-malarials who had repeat test- } \\
\text { ing after admission }\end{array}$ \\
\hline
\end{tabular}

(guideline dosage $\pm 20 \%$ ) were calculated. No attempts were made to impute data where these data were missing for calculating specific indicators (for example if the child's weight was missing correctness of drug dosage could not be estimated). In addition, the following indicators were used to assess the routine practice of using laboratory testing to guide management; proportion with laboratory requests of haemoglobin level, blood chemistry and glucose level. To identify evidence that clinicians considered other causes of febrile illnesses the proportion of malaria patients with the following tests requested: HIV status, blood culture/lumbar culture, x-rays and urine tests and the proportions prescribed antibiotics were calculated. All analyses were conducted using R statistical software (version 3.2.2) [19].

\section{Ethical consideration}

Scientific and ethical approval for the study was obtained from the Kenya Medical Research Institute Scientific and Ethics Review Unit as part of the CIN study (SSC protocol no. 2465) that provides for use of de-identified patient data obtained through retrospective review of medical records without individual informed consent for audit and quality improvement purposes.

\section{Results}

\section{Malaria diagnosis and clinical features}

During the 24-month period, there were 19,419 acute medical admissions out of which 13,014 (67\%) children had admission diagnosis of malaria (range 1597-4572 per hospital). The median age of malaria admissions across all hospitals was 24 months (interquartile range 12-36), and $53 \%$ were male. Fever was a common presenting feature, $91.6 \%$ of malaria admissions with complete documentation had fever (from 84.8 to $95.7 \%$ per hospital). Documentation of the specific guideline recommended signs of severe malaria (fever, pallor, altered consciousness level, respiratory distress, ability to drink and convulsions) within the medical records of malaria admissions ranged from 10 to $100 \%$ for the specific signs across hospitals (Fig. 1), and rapid improvements in documentation of malaria features was noted in three hospitals upon joining the CIN. Documentation of the five signs was $70 \%$ with a range of $47.2-83.6 \%$ across the hospitals. WHO defined severe malaria (characterized by the occurrence of danger signs of severe illness in a child with malaria diagnosis) was common with $49.4 \%$ of malaria admissions presenting with at least one danger sign. The specific danger signs for malaria were present in between 8.1 and $34.8 \%$ of admissions seen during the entire period: convulsions $(34.8 \%)$, respiratory distress (14.8\%), severe pallor (14.6\%), and altered level of consciousness (8.1\%) (Table 2).

\section{Investigation of admissions with clinical malaria}

Of all the 13,014 admissions with clinical malaria, 11,981 (92.1 \%) had a malaria test ordered during admission (range per hospital 82.5 to $98.7 \%$ ). Further, among these children with a malaria test requested 10,388/11,981 (86.7 \%) had malaria test results documented in the clinical notes (range per hospital 66.6-99.3\%).

The majority, 8050/10,388 (77.5\%) of documented malaria test results were positive for the malaria parasite. For those with negative malaria tests still treated presumptively, 52/1613 (3.2\%, hospitals' range 0-17.6\%), had malaria diagnostic tests repeated (Table 3 ). To guide management decisions in the children with malaria diagnosis $(\mathrm{N}=13,014)$ the following tests were done; 


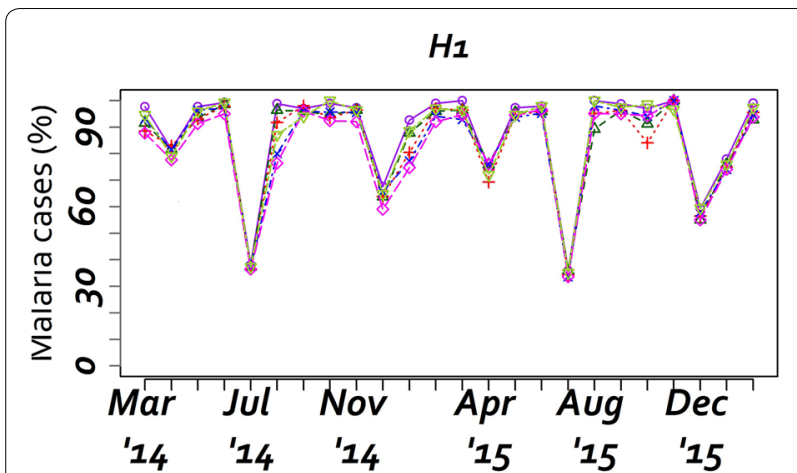

$H_{3}$

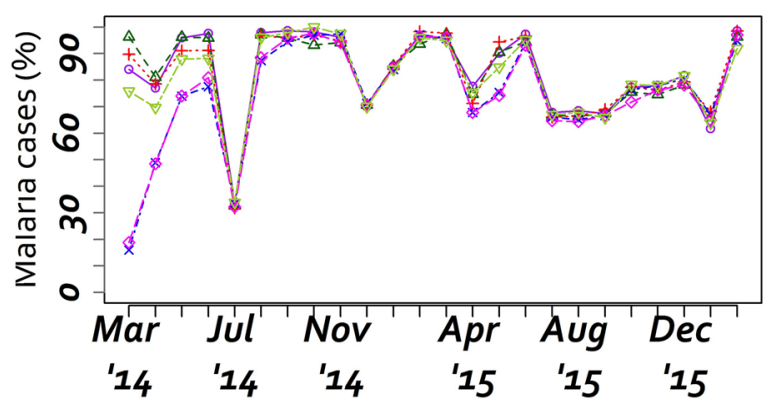

H8

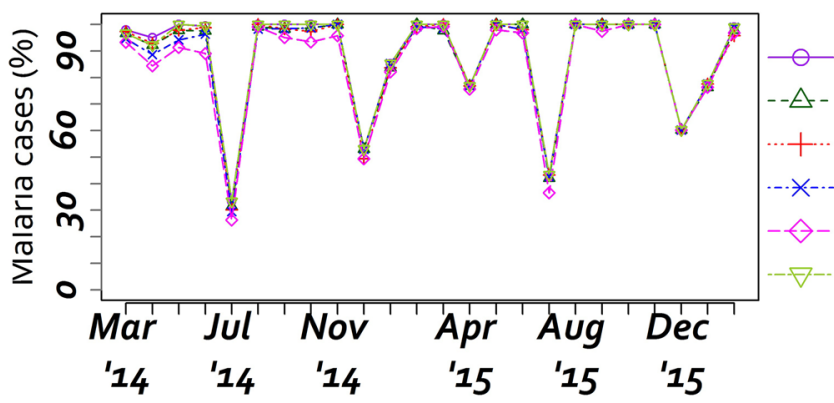

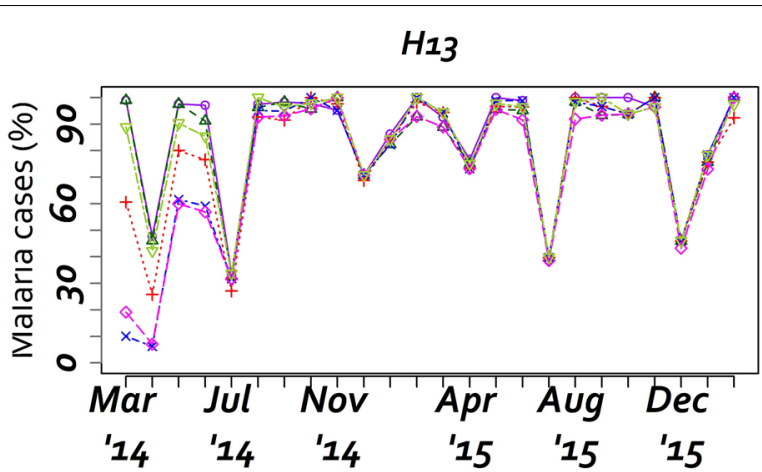

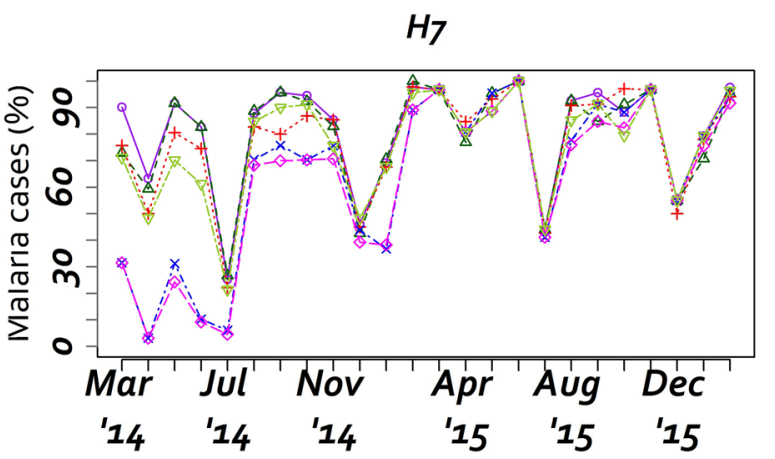

Fever

Pallor

Level of conciousness

Respiratory distress

Ability to drink

Convulsions

Fig. 1 Temporal trend in documentation of clinical signs from March, 2014 to February, 2016

haemoglobin level 4626 (35.5\%), blood chemistry 178 (1.4 \%) and blood glucose 333 (2.6\%). Among the children documented to have severe pallor, 1232/1583 (77.8 \%) had haemoglobin level checked. Other diagnostic tests done included: HIV status ascertained (39.6 \%), microbiology (blood culture or lumbar puncture for CSF culture and other microscopic and biochemical CSF investigations) $(8.5 \%)$, urine tests (1.9\%) and $\mathrm{x}$-rays (2.5\%).

\section{Treatment of malaria cases}

A total of $6745 / 8050$ (83.8 \%) patients with a positive malaria test were clearly prescribed anti-malarials. Among the remaining 1305 (16.2\%) a total of 1120/1305
$(85.8 \%)$ had no treatment information available. Of the 2338 patients with a negative diagnostic test, 1613 (69.0\%, range across hospitals 53.0-80.2\%) were prescribed anti-malarials out of which 1065 (66.0 \%) had signs or a diagnosis of severe malaria and only 37 (3.5\%) had a repeat test done with 28 (75.7 \%) being positive. There were 1033 (7.9\%) children who were not tested for malaria and of these 786 (76.1\%) had anti-malarials prescribed. The anti-malarial drugs and formulations used were tablets for oral artemether-lumefantrine and injections for quinine, artemether and artesunate. Among the cases with a positive malaria test given anti-malarials on admission $(\mathrm{n}=6745)$, quinine was the most prescribed drug in 3184 (47.2\%), followed by artesunate in 3108 
Table 2 Demographic characteristics and clinical signs of childhood malaria in children admitted to five Kenyan County Hospitals

\begin{tabular}{|c|c|c|c|c|c|c|}
\hline \multirow[t]{2}{*}{ Indicator } & \multirow[t]{2}{*}{ Overall } & \multicolumn{5}{|l|}{ Hospitals } \\
\hline & & H1 & H3 & H7 & $\mathrm{H} 8$ & $\mathrm{H} 13$ \\
\hline Number of malaria cases & 13014 & 2587 & 4572 & 1695 & 2563 & 1597 \\
\hline Male (\%) & $6880 / 13014(52.9)$ & $1388 / 2587(53.7)$ & $2430 / 4572(53.1)$ & $877 / 1695(51.7)$ & $1361 / 2563(53.1)$ & $824 / 1597(51.6)$ \\
\hline Age in months, median (IQR) & $24(12-36)$ & $20(12-36)$ & $23(12-36)$ & $20(11-36)$ & $15(9-27)$ & $24(12-36)$ \\
\hline All clinical features documented & $9104 / 13014(70.0)$ & $2013(77.8)$ & $3169(69.3)$ & $800(47.2)$ & $2143(83.6)$ & $979(61.3)$ \\
\hline Malaria admissions with fever ${ }^{\mathrm{a}}(\%)$ & 10063/10981 (91.6) & $2132 / 2255(94.5)$ & $3527 / 3854(91.5)$ & $1260 / 1317(95.7)$ & 1890/2229 (84.8) & $1254 / 1326(94.6)$ \\
\hline $\begin{array}{l}\text { Malaria admissions with at least one } \\
\text { danger sign }{ }^{b}(\%)\end{array}$ & $6427 / 13014(49.4)$ & $1412 / 2587(54.6)$ & $2204 / 4572(48.2)$ & $675 / 1695(39.8)$ & $1185 / 2563(46.2)$ & $951 / 1597(59.5)$ \\
\hline Altered level of consciousness (\%) & $863 / 10600(8.1)$ & 195/2160 (9.0) & $288 / 3828(7.5)$ & $74 / 1227(6.0)$ & $145 / 2201(6.6)$ & $161 / 1184(13.6)$ \\
\hline Inability to drink (\%) & $1264 / 9514(13.3)$ & $369 / 2111(17.5)$ & $244 / 3370(7.2)$ & $137 / 857(16.0)$ & 272/2121 (12.8) & 242/1055 (22.9) \\
\hline Respiratory distress (\%) & 1435/9692 (14.8) & 266/2161 (12.3) & 405/3373 (12.0) & $61 / 895(6.8)$ & $539 / 2185(24.7)$ & $164 / 1078(15.2)$ \\
\hline Severe pallor (\%) & 1583/10807 (14.6) & $383 / 2180$ (17.6) & 795/3848 (20.7) & 171/1281 (13.3) & $103 / 2202(4.7)$ & $131 / 1296(10.1)$ \\
\hline Convulsions (\%) & $3699 / 10625$ (34.8) & $775 / 2205(35.1)$ & 1227/3721 (33.0) & $427 / 1196(35.7)$ & $555 / 2223(25.0)$ & $715 / 1280(55.9)$ \\
\hline
\end{tabular}

$I Q R$ interquartile range

$\mathrm{a}, \mathrm{b}$ The denominator for proportion with clinical signs of malaria where the respective sign was documented

Table 3 Malaria testing and treatment practices among children admitted to five Kenyan County Hospitals: March, 2014 to February, 2016

\begin{tabular}{|c|c|c|c|c|c|c|}
\hline Indicator & Overall & $\mathrm{H} 1$ & $\mathrm{H} 3$ & $\mathrm{H7}$ & $\mathrm{H} 8$ & $\mathrm{H} 13$ \\
\hline Total malaria admissions $(\mathrm{N})$ & 13014 & 2587 & 4572 & 1695 & 2563 & 1597 \\
\hline \multicolumn{7}{|l|}{ Malaria investigations } \\
\hline $\begin{array}{l}\text { Proportion with malaria test } \\
\text { requested, } \mathrm{n} / \mathrm{N}(\%)\end{array}$ & $11981 / 13014(92.0)$ & 2533/2587 (97.9) & $3770 / 4572(82.5)$ & $1596 / 1695$ (94.2) & $2505 / 2563(97.7)$ & $1577 / 1597(98.7)$ \\
\hline Malaria test results available, n/N (\%) & 10388/11981 (86.7) & $2516 / 2533(99.3)$ & $2510 / 3770(66.6)$ & $1481 / 1596(92.8)$ & $2327 / 2505$ (92.9) & $1554 / 1577(98.5)$ \\
\hline $\begin{array}{l}\text { Positive malaria test in those with } \\
\text { results documented, n/N (\%) }\end{array}$ & $8050 / 10388(77.5)$ & $1902 / 2516(75.6)$ & $2046 / 2510(81.5)$ & $1162 / 1481(78.5)$ & $1609 / 2327(69.1)$ & $1331 / 1554(85.6)$ \\
\hline $\begin{array}{l}\text { Positive malaria test in all children } \\
\text { diagnosed with malaria, } \mathrm{n} / \mathrm{N}(\%)\end{array}$ & $8050 / 13014(61.9)$ & $1902 / 2587(73.5)$ & $2046 / 4572(44.8)$ & $1162 / 1695(68.6)$ & $1609 / 2563(62.8)$ & $1331 / 1597(83.3)$ \\
\hline $\begin{array}{l}\text { Diagnosis of severe malaria with } \\
\text { positive test, n/N (\%) }\end{array}$ & $5243 / 8050(65.1)$ & $1174 / 1902(61.7)$ & $1701 / 2046(83.1)$ & $699 / 1162(60.2)$ & $793 / 1609$ (49.3) & $876 / 1331(65.8)$ \\
\hline \multicolumn{7}{|l|}{ Antimalarial prescription } \\
\hline $\begin{array}{l}\text { Proportion with positive test pre- } \\
\text { scribed anti-malarial drug, n/N (\%) }\end{array}$ & $6745 / 8050(83.8)$ & 1642/1902 (86.3) & $1760 / 2046(86.0)$ & $909 / 1162(78.2)$ & $1347 / 1609(83.7)$ & 1087/1331 (81.7) \\
\hline $\begin{array}{l}\text { Proportion with negative test pre- } \\
\text { scribed anti-malarial drug, n/N (\%) }\end{array}$ & $1613 / 2338(69.0)$ & $479 / 614(78.0)$ & $372 / 464(80.2)$ & $169 / 319(53.0)$ & $445 / 718(62.0)$ & $148 / 223(66.4)$ \\
\hline $\begin{array}{l}\text { Proportion of negative test treated } \\
\text { presumptively with repeat testing, } \\
\mathrm{n} / \mathrm{N}(\%)\end{array}$ & $52 / 1613(3.2)$ & $7 / 479(1.5)$ & 0 & $1 / 169(0.6)$ & $18 / 445(4.0)$ & $26 / 148(17.6)$ \\
\hline $\begin{array}{l}\text { Proportion with no malaria test } \\
\text { treated presumptively, n/N (\%) }\end{array}$ & $786 / 1033(76.1)$ & $28 / 54(51.9)$ & $674 / 802(84.0)$ & $45 / 99(45.5)$ & $35 / 58(60.3)$ & $4 / 20(20.0)$ \\
\hline \multicolumn{7}{|l|}{ Antibiotic prescription } \\
\hline $\begin{array}{l}\text { Proportion of all malaria admission } \\
\text { cases prescribed antibiotics }{ }^{a}, \mathrm{n} / \mathrm{N} \\
(\%)\end{array}$ & $6966 / 13014(53.5)$ & $1428 / 2587(55.2)$ & $2615 / 4572(57.2)$ & $609 / 1695$ (35.9) & $1555 / 2563(60.7)$ & $759 / 1597(47.5)$ \\
\hline $\begin{array}{l}\text { Proportion with positive test pre- } \\
\text { scribed antibiotics }{ }^{\mathrm{a}}, \mathrm{n} / \mathrm{N}(\%)\end{array}$ & $4041 / 8050(50.2)$ & 1025/1902 (53.9) & $1122 / 2046(54.8)$ & $369 / 1162(31.8)$ & $920 / 1609(57.2)$ & $605 / 1331(45.5)$ \\
\hline $\begin{array}{l}\text { Proportion with negative prescribed } \\
\text { antibiotics }{ }^{a}, \mathrm{n} / \mathrm{N}(\%)\end{array}$ & $1463 / 2338(62.6)$ & $368 / 614(59.9)$ & $290 / 464(62.5)$ & 172/319 (53.9) & $497 / 718(69.2)$ & $136 / 223(61.0)$ \\
\hline
\end{tabular}

\footnotetext{
a Broad spectrum antibiotics including crystalline penicillin, gentamicin, ceftriaxone, amoxicillin or chloramphenicol was prescribed
} 
(46.1\%) and the least prescribed drug on admission was artemether-lumefantrine in 250 (3.7 \%) (Table 4). Hospitals most commonly prescribed parenteral quinine except H8 where 89.0 \% were prescribed artesunate (see Fig. 2). For children with a negative malaria test given antimalarials $(\mathrm{n}=1613)$, artesunate was the most prescribed drug 845 (52.4 \%) followed by quinine 597 (37.0\%).

Among all malaria cases who were prescribed quinine, $59.1 \%$ of the loading and $93.1 \%$ of the maintenance doses were correct. Approximately two-thirds (63.8\%) of children given artesunate were prescribed the recommended dose.

Prescription patterns of anti-malarial drugs varied by hospital and by time (Fig. 2). In H1 and H3 they alternated between quinine and artesunate. Prescription of artesunate was consistently high in $\mathrm{H} 8$, whereas $\mathrm{H} 3$, $\mathrm{H} 7$ and $\mathrm{H} 13$ transitioned to use of artesunate during the monitoring period. Among the cases who were prescribed either quinine or artesunate, $(\mathrm{n}=9840), 7598$ (77.2 \%) had signs suggestive of severe or complicated malaria or a diagnosis of severe malaria. Anti-malarial prescription rates for children with a negative test ranged between 15 and $100 \%$ across the hospitals throughout the period. Antibiotics prescription among the patients testing negative and positive for malaria was 62.6 and $50.2 \%$, respectively (Table 3 ).

\section{Discussion}

The aim of this study was to determine the investigation and treatment practices for children aged 2-59 months admitted with malaria in Western Kenya County hospitals 3 and 4 years after national and international malaria guidelines changed respectively. Documentation of clinical features of malaria (whether present or absent) was good, including the signs that define severe malaria; level of consciousness and respiratory distress [10]. This is an improvement compared to an earlier studies done in Kenya [20], Uganda [21] and in Benin [22], and is likely due to routine use of a structured Paediatric Admission Record (PAR) that guides junior clinicians to conduct a complete physical examination of sick children at admission $[23,24]$ associated with feedback on documentation quality given to hospitals in the form of two monthly reports [17]. Malaria laboratory testing rates when commencing inpatient treatment for clinical malaria were $92 \%$ with recording of diagnostic test results in medical notes found in over three quarters. Findings on rates of testing and reporting are similar to some recent studies in Uganda [25] and Benin [22], but better than other studies from the region and earlier studies from Kenya [20, 23, 26-28]. Findings therefore suggest some success in adoption of the test and treat approach.

Over $80 \%$ of malaria positive cases were clearly prescribed anti-malarial drugs. However, almost half were prescribed intravenous quinine despite the change in recommendations to use of artesunate in 2012. The trend was consistent across all the hospitals in the beginning of the period except $\mathrm{H} 8$ where most patients were given artesunate throughout the study period. In addition, there was a shift towards intravenous artesunate towards the end of the period in H3, H7 and H13. Similar results were reported in Uganda [25] and although the use of artesunate in 2014/2016 is unsatisfactory it is an improvement on a 2012 Kenyan survey when no inpatient malaria patients were given artesunate [23]. Informal enquiries through hospital staff indicated that the consistent prescription of artesunate in $\mathrm{H} 8$ was because of local efforts to ensure consistent drug availability (including local purchase). The lower rates of prescribing artesunate in other facilities were in part attributed to stock outs as well as continued difficulties changing prescribing habits.

While there is a transition from quinine to artesunate almost half of the clinical malaria cases prescribed parenteral anti-malarials had no signs of severe malaria documented (and thus were able to eat and drink). Despite

Table 4 Anti-malarial drugs prescription in children with a positive malaria test across 5 Kenyan County Hospitals

\begin{tabular}{|c|c|c|c|c|c|c|}
\hline \multirow{2}{*}{$\begin{array}{l}\text { Type of anti- } \\
\text { malarial }\end{array}$} & \multirow{2}{*}{$\begin{array}{l}\text { Overall }(n=6745) \\
(\%)\end{array}$} & \multicolumn{5}{|l|}{ Hospitals } \\
\hline & & $H 1(n=1642)(\%)$ & H3 $(n=1760)(\%)$ & H7 (n= 909) (\%) & $H 8(n=1347)(\%)$ & H13 $(n=1087)(\%)$ \\
\hline Quinine & $3184(47.2)$ & $1075(65.5)$ & $946(53.8)$ & $587(64.9)$ & $13(1.0)$ & $563(51.8)$ \\
\hline Artesunate & $3108(46.1)$ & $478(29.1)$ & $739(42.0)$ & $244(26.8)$ & 1199 (89.0) & $448(41.2)$ \\
\hline $\begin{array}{l}\text { Quinine and artesu- } \\
\text { nate }\end{array}$ & $158(2.3)$ & $56(3.4)$ & $26(1.5)$ & $54(5.9)$ & $2(0.2)$ & $20(1.8)$ \\
\hline $\begin{array}{l}\text { Coartem } \\
\text { (Artemether-lume- } \\
\text { fantrine) }\end{array}$ & $250(3.7)$ & $33(2.0)$ & $18(1.0)$ & $19(2.1)$ & $124(9.2)$ & $56(5.2)$ \\
\hline Other anti-malarials ${ }^{\mathrm{a}}$ & $45(0.7)$ & 0 & $31(1.8)$ & $5(0.6)$ & $9(0.7)$ & 0 \\
\hline
\end{tabular}

a Either a combination of artesunate or quinine or artemether lumefantrine with artemether or artemether only prescribed 


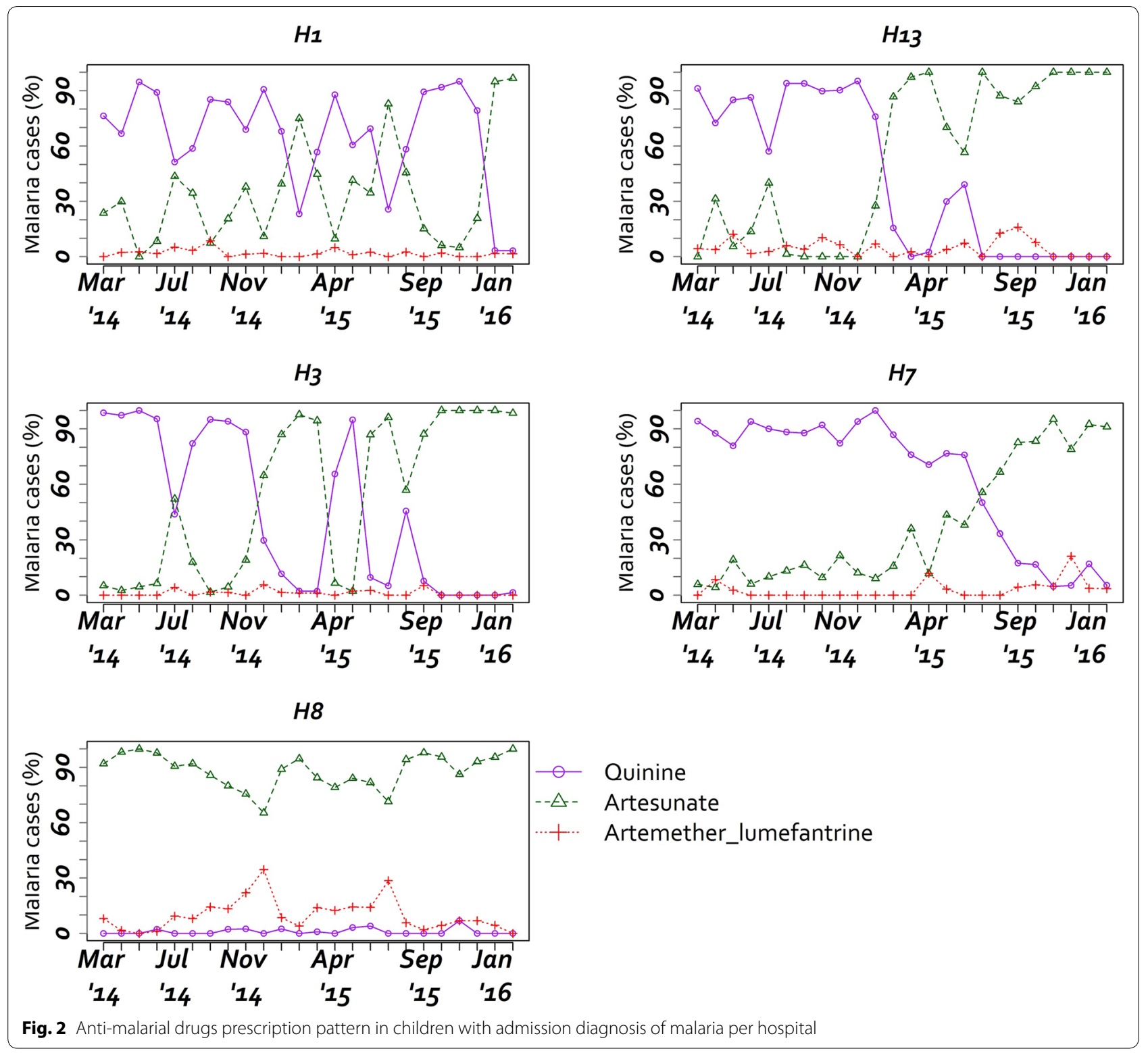

this there were very low rates of giving the recommended ACT, AL to these 'non-severe' cases, signifying potential overuse of injectable anti-malarials in children [21]. This may be attributed to clinicians' preference for parenteral drugs and the belief that they are 'stronger' [29]. On a positive note most of the dosing per body weight of both quinine and artesunate was correct, similar to a recent study in Uganda [21], but representing a considerable improvement from earlier Kenyan studies and potentially attributable to efforts to promote national paediatric guidelines in Kenya [26].

These data show high rates of presumptive treatment among children admitted with a negative malaria test or without a test done. Some reasons for this might include; associating malaria with high mortality [30], mistrust of negative test results, and concerns about missing possible malaria cases with potentially fatal consequences [30, 31]. Similar results have been reported in various studies across Africa [20, 21, 28, 30, 32-34]. The trend seems to be different from outpatient studies done immediately after the 2010 new treatment guidelines in Uganda [35] and Tanzania [36], and recent findings, that reported a decrease in prescribing anti-malarials to malaria test negative patients in Kenya [13]. Worth noting is the low rate of repeat testing of negative cases that were started on anti-malarials despite the national guidelines' 
recommendation of repeat testing to confirm absence of malaria and stopping treatment if the results are still negative [10,37]. This 'over-treatment' of malaria might lead to missing potentially treatable alternative causes of febrile illness with a negative malaria test that has been associated with high mortality [38].

As clinical features of malaria often overlap with other causes of febrile illness in children [39] or there might be co-infection with other diseases [40], it is important that health systems can support and that clinicians perform other laboratory investigations to identify and treat other causes of disease. Of some concern is the low use or availability of blood glucose testing in this population given that hypoglycaemia is common. The findings also revealed infrequent use in most hospitals of other tests (x-rays, microbiology tests, HIV test) to identify other causes of severe febrile illness in children admitted with clinical malaria. Anecdotally inability to conduct blood culture or lumbar puncture is a common problem underlying limited use of these investigations.

The study had some limitations. Firstly, the inclusion of patients in the analysis depended on the clinicians' diagnosis of malaria, and further analysis relied on their documentation of testing and laboratory records. Similarly, the study relied on documentation in the case record of symptoms, signs and treatments to examine practices. The potential challenges of using such routine information that often has missing data were minimised through use of standardized record forms to record clinical information, timing data collection during discharge, data quality checks implemented within CIN, feedback to and development of partnerships with hospitals in the CIN all of which improved data availability and quality [17]. The study only captures data from five hospitals, hence generalizing results to other facilities in Kenya should be done with caution. Lastly, data on treatment preceding hospitalization were not available. Conceivably these information may have influenced some clinical decisions and practices which we assessed. The potential threat to reported findings posed by preadmission investigations not recorded in the CIN data is minimal because from our experience clinicians rarely use investigations conducted outside their facility as a basis for treatment for acute medical illnesses because these investigations are often perceived as unreliable, and also the essential investigations are readily available within facilities at minimal costs or for diseases like malaria these investigations are provided at no cost to patients. Despite these limitations, the findings from this study can provide an insight into the quality of routine inpatient malaria case management and the extent to which national and international policies are influencing practice.

\section{Conclusions}

There is evidence of improvements in malaria case management over the initial 24 months of Clinical Information Network activities but presumptive malaria treatment for test negative results, and overuse of injectable instead of oral anti-malarials are still common practices. Three years after national policy change of the severe malaria first-line treatment to injectable artesunate, there is a gradual transition to use of artesunate. The findings suggest continued need for improvement in dissemination and implementation of guidelines for inpatient care and improved, reliable access to recommended investigations and drugs. Finally, efforts to improve clinical information could help track progress.

\section{Authors' contributions}

BA, PA, MO, TJ, GI and ME helped support analyses, draft the initial report and contributed to its development and approved the final version. All authors read and approved the final manuscript.

\section{Author details}

${ }^{1}$ Kenya Medical Research Institute/Wellcome Trust Research Programme, P.O. Box 43640, Nairobi 00100, Kenya. ${ }^{2}$ Department of Paediatrics and Child Health, University of Nairobi, Nairobi, Kenya. ${ }^{3}$ Nuffield Department of Medicine, Oxford University, Oxford, UK.

\section{Acknowledgements}

The authors would like to thank the Ministry of Health who gave permission for this work to be developed and have supported the implementation of the Clinical Information Network (CIN) together with the county health executives and all hospital management teams. They are grateful to the Kenya Paediatric Association for promoting the aims of the CIN and the support they provided through their officers and membership. They also thank the hospital clinical teams on all the paediatric wards who provide care to the children for whom this project is designed.

The Clinical Information Network author group, which contributed to the conduct of the work, collection of data and data quality assurance, includes Samuel Ng'arng'ar (Vihiga County Hospital), Magdalene Kuria (Kisumu East County Hospital), Barnabas Kigen (Busia County Hospital), Jedida Chepkorir and Nick Aduro (Kakamega County Hospital) and Rachel Inginia and Melab Musabi (Kitale County Hospital).

\section{Competing interests}

The authors declare that they have no competing interests when conducting the research.

\section{Availability of data and material}

Data for this report are under the primary jurisdiction of the Ministry of Health in Kenya. Enquiries about using the data can be made to the KEMRI-Wellcome Trust Research Programme Data Governance Committee.

\section{Consent for publication}

This work is published with the permission of the Director of KEMRI.

\section{Ethics approval}

Kenya Medical Research Institute Scientific and Ethics Review Unit approved the study as part of the CIN study (SSC protocol no. 2465).

\section{Funding}

Funds from The Wellcome Trust (\#097170) awarded to ME as a Senior Fellowship together with additional funds from a Wellcome Trust core grant awarded to the KEMRI-Wellcome Trust Research Programme (\#092654) supported this work. The funders had no role in drafting or submitting this manuscript.

Received: 25 June 2016 Accepted: 5 October 2016

Published online: 18 October 2016 


\section{References}

1. WHO. World Malaria Report 2014. Geneva: World Health Organization; 2014

2. WHO. Management of severe malaria: a practical handbook. 3rd ed. Geneva: World Health Organization; 2012.

3. Allen SJ, O'Donnell A, Alexander ND, Clegg JB. Severe malaria in children in Papua New Guinea. QJM. 1996:89:779-88.

4. English M, Punt J, Mwangi I, McHugh K, Marsh K. Clinical overlap between malaria and severe pneumonia in Africa children in hospital. Trans R Soc Trop Med Hyg. 1996;90:658-62.

5. Marsh K, Forster D, Waruiru C, Mwangi I, Winstanley M, Marsh V, et al. Indicators of life-threatening malaria in African children. N Engl J Med. 1995;332:1399-404

6. Waller D, Krishna S, Crawley J, Miller K, Nosten F, Chapman D, et al. Clinical features and outcome of severe malaria in Gambian children. Clin Infect Dis. 1995:21:577-87.

7. Dondorp AM, Fanello Cl, Hendriksen IC, Gomes E, Seni A, Chhaganlal KD et al. Artesunate versus quinine in the treatment of severe falciparum malaria in African children (AQUAMAT): an open-label, randomised trial. Lancet. 2010;376:1647-57.

8. WHO. Guidelines for the treatment of malaria. Geneva: World Health Organization; 2010

9. WHO. Guidelines for the treatment of malaria. 3rd ed. Geneva: World Health Organization; 2015.

10. Ministry of Public Health and Sanitation \& Ministry of Medical Services. National guidelines for diagnosis, treatment and prevention of malaria in Kenya. Division of Malaria Control. Nairobi, Kenya, 2010.

11. Ministry of Public Health and Sanitation. National guidelines for diagnosis, treatment and prevention of malaria for health workers. Fourth edition. Nairobi, Kenya, 2012.

12. Nyandigisi A, Memusi D, Mbithi A, Ang'wa N, Shieshia M, Muturi A, et al. Malaria case-management following change of policy to universal parasitological diagnosis and targeted artemisinin-based combination therapy in Kenya. PLoS ONE. 2011;6:e24781.

13. Zurovac D, Githinji S, Memusi D, Kigen S, Machini B, Muturi A, et al. Major improvements in the quality of malaria case-management under the "Test and Treat" policy in Kenya. PLoS ONE. 2014;9:e92782.

14. Chandler Cl, Chonya S, Boniface G, Juma K, Reyburn H, Whitty CJ. The importance of context in malaria diagnosis and treatment decisions-a quantitative analysis of observed clinical encounters in Tanzania. Trop Med Int Health. 2008;13:1131-42.

15. Chandler Cl, Mwangi R, Mbakilwa H, Olomi R, Whitty CJ, Reyburn H. Malaria overdiagnosis: is patient pressure the problem? Health Policy Plan. 2008:23:170-8

16. Chauhan D, Mason A. Factors affecting the uptake of new medicines in secondary care - a literature review. J Clin Pharm Ther. 2008;33:339-48. doi:10.1111/j.1365-2710.2008.00925.x

17. Tuti T, Bitok M, Paton C, Makone B, Malla L, Muinga N, et al. Innovating to enhance clinical data management using non-commercial and open source solutions across a multi-center network supporting inpatient pediatric care and research in Kenya. J Am Med Inform Assoc. 2015;23:184-92

18. English M. Designing a theory-informed, contextually appropriate intervention strategy to improve delivery of paediatric services in Kenyan hospitals. Implement Sci. 2013:8:39. doi:10.1186/1748-5908-8-39.

19. R Development Core Team. R: a language and environment for statistical computing. Vienna, Austria: R Foundation for Statistical Computing: 2015.

20. English M, Esamai F, Wasunna A, Were F, Ogutu B, Wamae A, et al. Assessment of inpatient paediatric care in first referral level hospitals in 13 districts in Kenya. Lancet. 2004;363:1948-53.

21. Achan J, Tibenderana J, Kyabayinze D, Mawejje H, Mugizi R, Mpeka B, et al. Case management of severe malaria-a forgotten practice: experiences from health facilities in Uganda. PLoS ONE. 2011;6:e17053.
22. Mace KE, Gueye AS, Lynch MF, Tassiba EM, Rowe AK. An evaluation of methods for assessing the quality of case management for inpatients with malaria in Benin. Am J Trop Med Hyg. 2014;91:354-60.

23. Gathara D, Nyamai R, Were F, Mogoa W, Karumbi J, Kihuba E, et al. Moving towards routine evaluation of quality of inpatient pediatric care in Kenya. PLOS ONE. 2015;10:e0117048.

24. Ayieko P, Ntoburi S, Wagai J, Opondo C, Opiyo N, Migiro S, et al. A multifaceted intervention to implement guidelines and improve admission paediatric care in Kenyan district hospitals: a cluster randomised trial. PLOS Med. 2011;8:e1001018.

25. Sserwanga A, Sears D, Kapella BK, Kigozi R, Rubahika D, Staedke SG, et al. Anti-malarial prescription practices among children admitted to six public hospitals in Uganda from 2011 to 2013. Malar J. 2015;14:331.

26. English M, Gathara D, Mwinga S, Ayieko P, Opondo C, Aluvaala J, et al. Adoption of recommended practices and basic technologies in a lowincome setting. Arch Dis Child. 2014:99:452-6.

27. Fenny AP, Hansen KS, Enemark U, Asante FA. Quality of uncomplicated malaria case management in Ghana among insured and uninsured patients. Int J Equity Health. 2014;13:63.

28. Mbonye MK, Burnett SM, Naikoba S, Colebunders R, Wouters K, Weaver $M R$, et al. Malaria care in infants aged under 6 months in Uganda: an area of unmet needs! PLOS ONE. 2015;10:e0123283.

29. Li HK, Agweyu A, English M, Bejon P. An unsupported preference for intravenous antibiotics. PLoS Med. 2015;12:e1001825.

30. Onchiri FM, Pavlinac PB, Singa BO, Naulikha JM, Odundo EA, Farquhar $C$, et al. Frequency and correlates of malaria over-treatment in areas of differing malaria transmission: a cross-sectional study in rural Western Kenya. Malar J. 2015;14:97

31. Odaga J, Sinclair D, Lokong JA, Donegan S, Hopkins H, Garner P. Rapid diagnostic tests versus clinical diagnosis for managing people with fever in malaria endemic settings. Cochrane Database Syst Rev. 2014;7:8998.

32. Bouyou-Akotet MK, Mawili-Mboumba DP, Kendjo E, Eyang Ekouma A, Abdou Raouf O, Engohang Allogho E, et al. Complicated malaria and other severe febrile illness in a pediatric ward in Libreville, Gabon. BMC Infect Dis. 2012;12:216

33. Moon AM, Biggs HM, Rubach MP, Crump JA, Maro VP, Saganda W, et al. Evaluation of in-hospital management for febrile illness in Northern Tanzania before and after 2010 World Health Organization Guidelines for the treatment of malaria. PLoS ONE. 2014;9:e89814.

34. Reyburn H, Mbatia R, Drakeley C, Carneiro I, Mwakasungula E, Mwerinde $\mathrm{O}$, et al. Overdiagnosis of malaria in patients with severe febrile illness in Tanzania: a prospective study. BMJ. 2004;329:1212

35. Sserwanga A, Harris JC, Kigozi R, Menon M, Bukirwa H, Gasasira A, et al. Improved malaria case management through the implementation of a health facility-based sentinel site surveillance system in Uganda. PLoS ONE. 2011:6:e0016316.

36. Bastiaens GJ, Schaftenaar E, Ndaro A, Keuter M, Bousema T, Shekalaghe SA. Malaria diagnostic testing and treatment practices in three different Plasmodium falciparum transmission settings in Tanzania: before and after a government policy change. Malar J. 2011;10:76.

37. Ministry of Health. Basic pediatric protocols. Nairobi: Ministry of Health Government of Kenya; 2012

38. Evans J, Adusei A, Timmann C, May J, Mack D, Agbenyega T, et al. High mortality of infant bacteraemia clinically indistinguishable from severe malaria. QJM. 2004:97:591-7.

39. Chandramohan D, Jaffar S, Greenwood B. Use of clinical algorithms for diagnosing malaria. Trop Med Int Health. 2002;7:45-52.

40. Berkley JA, Mwangi I, Mellington F, Mwarumba S, Marsh K. Cerebral malaria versus bacterial meningitis in children with impaired consciousness. QJM. 1999:92:151-7. 